\title{
COMMUNICABLE DISEASES, NSW: MARCH 2002
}

\section{TRENDS}

Through to January 2002 relatively few cases of Ross River virus infections were reported across the state for the time of year. Barmah Forest virus infections were predominantly notified from the north coast of NSW (Tables 1 and 2, Figure 1).

We are happy to report that there were no cases of measles reported in the previous three months (Figure 1).

We are also happy to report that, after a prolonged epidemic, notifications of pertussis seem to be declining. Nonetheless, there are still many cases about, with 391 notifications being received in January. To protect vulnerable members of the community, vigilance is important in terms of ensuring complete immunisation of children, case finding, treatment, and prophylaxis of household contacts with erythromycin.

\section{MENINGOCOCCAL DISEASE AMONG CRUISE SHIP PASSENGERS}

On Friday 25 January 2002, the South Western Sydney Public Health Unit (SWSPHU) notified the NSW Department of Health's Communicable Diseases Unit (CDU) of the death of a 21-year-old South Western Sydney man from suspected invasive meningococcal disease. The man was taken by ambulance to hospital on 24 January after collapsing at his home. He had a three-day history of sore throat but had otherwise been well. A rash was noted and a diagnosis of meningococcal disease was made. Despite aggressive intervention, the man died.

In the seven days prior to the onset of his illness, the man had been on a cruise to the South Pacific. The cruise ship carried over a thousand passengers from all over Australia.

SWSPHU identified over 50 close contacts of the man who may have been at increased risk of disease, and provided them with information about the disease and with antibiotics to help prevent its further spread. The CDU informed local public health units and other states and territories about the case. Shortly after, the South Australian Department of Health reported that a South Australian man on the same cruise had been diagnosed with meningococcal disease on 22 January 2002. The man's close contacts had been contacted and given antibiotics.

No direct personal link between the cases was established. The cruise operator agreed to contact all passengers and crew from the ship to tell them about these events and about meningococcal disease. The NSW Department of Health set up a hotline providing general information to the public, issued media releases, and conducted regular media interviews to update the public on events. Passengers were alerted to seek medical attention if they develop symptoms of the disease. As a result of the public warnings, several other passengers were investigated for possible meningococcal infection, but in none of these was the diagnosis confirmed.

\section{HEPATITIS A INCREASING}

Notifications of hepatitis A reached a nadir of seven in April 2001, but have since increased. Thirty cases had onset in December 2001. Of these 30 cases, the biggest reported exposure was male-to-male sex (nine cases or 30 per cent), followed by overseas travel and eating at restaurants (five cases each or 17 per cent each). By area of residence, male-to-male sex is the most prominently reported exposure in South Eastern Sydney and Central Sydney. Men who have sex with men are at increased risk of hepatitis A, which is acquired through faecal-oral contact, including during sexual activity. Outbreaks of hepatitis A have been recorded among men who have sex with men in South Eastern Sydney every few years, most recently in $1998 .^{1}$

Hepatitis A can be prevented through:

- careful hand-washing with soap and running water after using the toilet, before handling food, and before and after sex;

- avoidance of exposure to faecal material;

- administration of immunoglubulin, which is recommended for household and other close contacts of cases;

- immunisation, which is recommended for some people at increased risk of the disease, including men who have sex with men.

Clinicians should be alert to the possible diagnosis of hepatitis A, especially among men who have sex with men living in or visiting Sydney, and notify the local public health unit (listed under 'Health' in the White Pages) of cases. PHU staff will help investigate the likely source and to help prevent disease in contacts.

\section{CRYPTOSPORIDIOSIS}

Notifications of cryptosporidiosis increased in December (21 cases) and January (30 cases). Most cases were individuals who resided in rural areas. About half the cases were children under five years of age. No common source has been identified among cases.

Cryptosporidiosis is a diarrhoeal illness caused by a waterborne parasite. Infections have been linked to drinking water, recreational water, childcare settings, person-toperson and animal-to-person contact. Large outbreaks of cryptosporidiosis have been recorded every three or four years in NSW, the last in 1997-8, associated with swimming in contaminated swimming pools., ${ }^{2,3}$

To avoid catching cryptosporidiosis: 
- always wash hands thoroughly with soap and running water after using the toilet, handling, animals, changing nappies, or working in the garden;

- always wash hands thoroughly before preparing food and drinks;

- do not drink untreated water (for example, from rivers, streams, lakes and dams). Boiling water from these sources for one minute will kill germs, including cryptosporidiosis.

To avoid spreading cryptosporidiosis:

- keep small children who have diarrhoea home from school, preschool, childcare or playgroups until the diarrhoea has completely stopped;

- food handlers, childcare workers, and health care workers with cryptosporidiosis should not work until diarrhoea has stopped;

- do not use swimming pools or other water recreational areas, or share linen and towels with others, for at least one week after the diarrhoea has stopped.
Swimming pool operators should follow the NSW Department of Health's Protocol for Minimising the Risk of Cryptosporidium in Public Swimming Pools and Spa Pools. This is available from the Department's web site at www.hprb.health.nsw.gov.au/public-health/ehb/general/ pools/publicpools.html.

Clinicians should consider the diagnosis among people presenting with diarrhoea lasting more than a few days. If suspected, the diagnosis should be confirmed with a stool sample specifically requesting a test for Cryptosporidium.

\section{REFERENCES}

1. Delpech V, Habib M, Lin M, et al. Hepatitis A in New South Wales, 1991-2000. NSW Public Health Bulletin 2002. In press.

2. Puech MC, McAnulty JM, Lesjak M, et al. A statewide outbreak of cryptosporidiosis in NSW associated with swimming at public pools. Epidemiol Infect 2001; 126:389396.

3. Lemmon JM, McAnulty JM, Bawden Smith J. Outbreak of cryptosporidiosis linked to an indoor swimming pool. Med $J$ Aust 1996; 165(2): 613-616. 骂 
FIGURE 1

\section{REPORTS OF SELECTED COMMUNICABLE DISEASES, NSW, JANUARY 1996 TO MAY 2001,} BY MONTH OF ONSET

These are preliminary data: case counts for recent months may increase because of reporting delays. Laboratory-confirmed cases, except for measles, meningococcal disease and pertussis. actual _ _ _ predicted after adjusting for likely reporting delays.

\begin{tabular}{|rc|}
\hline \multicolumn{3}{|c|}{ NSW population } \\
Male & $50 \%$ \\
$<5$ & $7 \%$ \\
$5-24$ & $28 \%$ \\
$25-64$ & $52 \%$ \\
$65+$ & $13 \%$ \\
Rural $^{*}$ & $42 \%$ \\
\hline
\end{tabular}
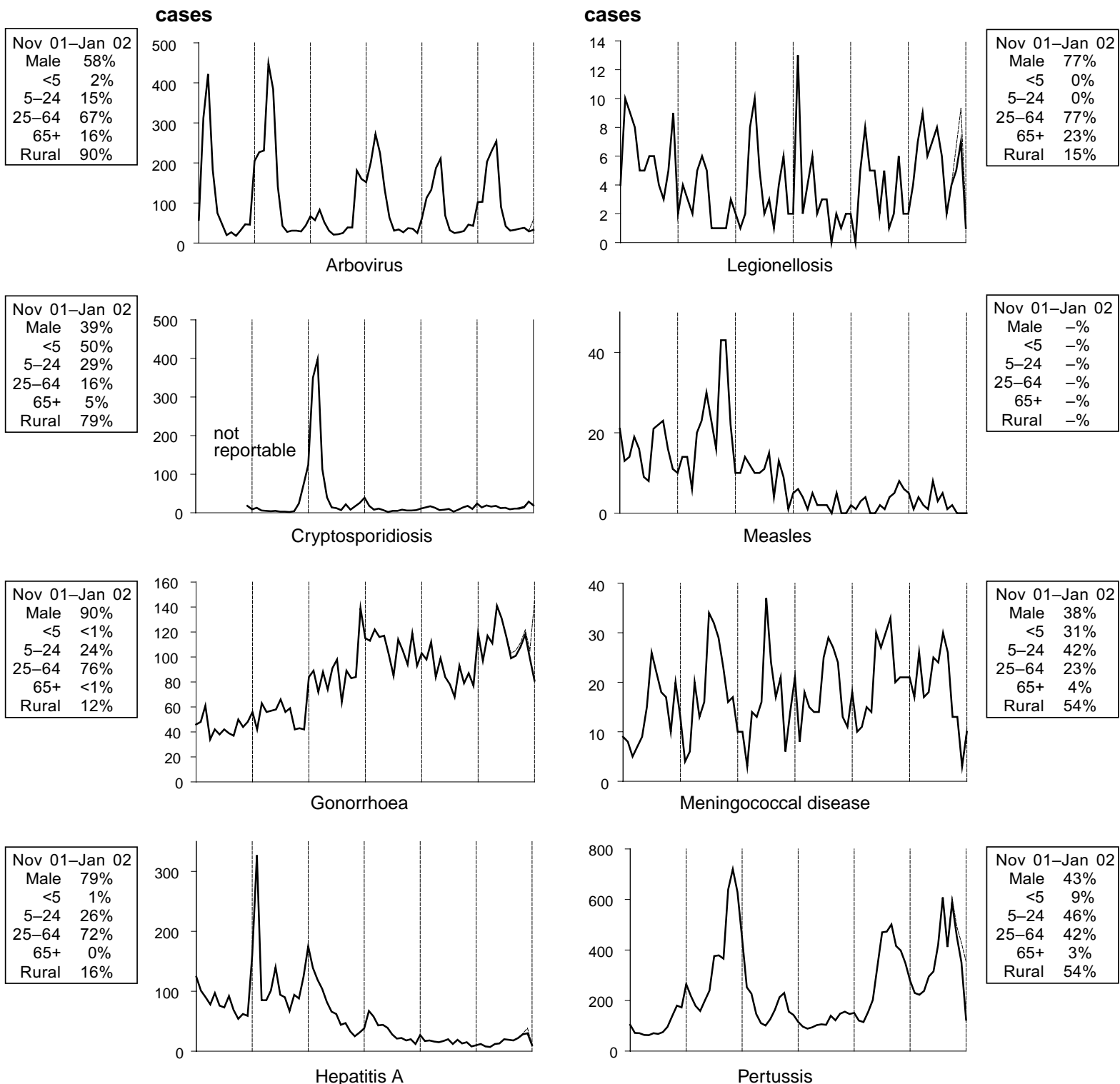

Hepatitis A
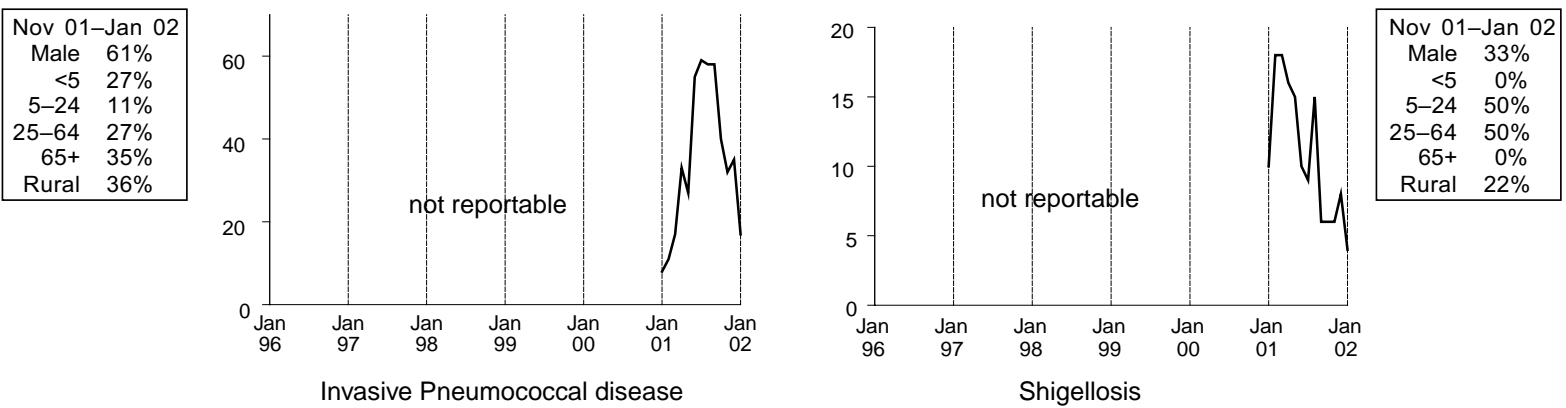

* For definition, see NSW Public Health Bulletin, April 2000 


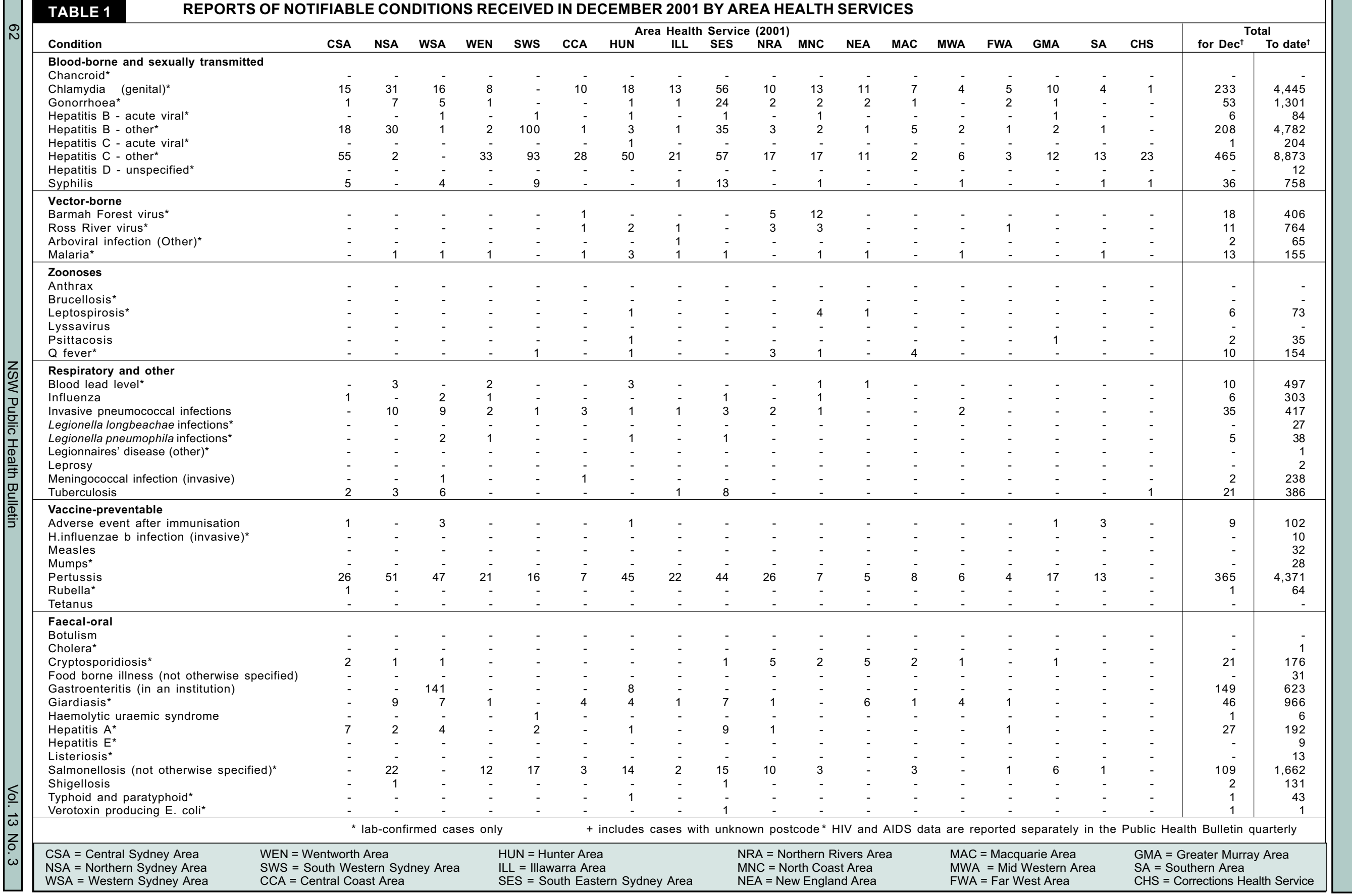


Gonorrhoea

Hepatitis B - acute viral*

Hepatitis B - other

Hepatitis C - other*

Hepatitis D - unspecified*

Syphilis

$\begin{array}{rrrrrrrr}53 & 27 & 40 & - & - & - & - & - \\ 17 & 12 & 9 & 1 & 3 & 5 & 27 & 14 \\ 1 & 3 & & - & 1 & 5 & 3 & 3\end{array}$

Vector-borne

Barmah Forest virus

Ross River virus ${ }^{*}$

Arboviral infection (Other)

Malaria

Zoonoses

Brucellosis*

Leptospirosis

Lyssavirus

Q fever*

Respiratory and other

Blood lead level

Invasive

Legionella longococcal infections

作

Legionnaires' disease (other)*

Leprosy

Meningococcal infection (invasive)

Vaccine-preventable

Vaccine-preventable

Adverse event after immunisation
H.influenzae $b$ infection (invasive)

Mumps*es

Pertussis

Pertussis
Rubella*

Rubella*
Tetanus

Faecal-oral

Botulism

Cholera $^{*}$
Cryptosporidiosis*

Food borne illness (not otherwise specified)

Gastroenteritis (in an institution)

Giardiasis*

Haemolytic uraemic syndrome

Hepatitis $A^{*}$
Hepatitis $E^{*}$

Listeriosis*

Salmonellosis (not otherwise specified)*

Shigellosis

Typhoid and paratyphoid ${ }^{\star}$

Verotoxin producing E. coli 